\title{
2 Generation of a mitochondrial protein compendium in Dictyostelium discoideum
}

Anna V Freitas ${ }^{1,3}$, Jake T Herb ${ }^{1,3}$, Miao Pan ${ }^{2}$, Yong Cheng ${ }^{1}$, Marjan Gucek ${ }^{1}$, Tian Jin $^{2}$ and Hong Xu ${ }^{1}$

${ }^{1}$ National Heart, Lung and Blood Institute, ${ }^{2}$ National Institute of Allergy and Infectious Diseases,

7 National Institute of Health, 9000 Rockville Pike, Bethesda, MD 20892

$8{ }^{3}$ These authors contributed equally to this work

9 Correspondence to: Hong Xu (hong.xu@nih.gov)

\section{Abstract}

The social amoeba Dictyostelium discoideum is a well-established model to study numerous cellular processes including cell motility, chemotaxis, and differentiation. As energy metabolism is involved in these processes, mitochondrial genetics and bioenergetics are of interest, though many features of Dictyostelium mitochondria differ from metazoans. A comprehensive inventory of mitochondrial proteins is critical to understanding mitochondrial processes and their involvement in various cellular pathways. Here, we utilized high-throughput multiplexed protein quantitation and homology analyses to generate a high-confidence mitochondrial protein compendium. Our proteomic approach, which utilizes quantitative mass spectrometry in combination with mathematical modeling, was validated through mitochondrial targeting sequence prediction and live-cell imaging. Our final compendium consists of 1082 proteins. Within our $D$. discoideum mitochondrial proteome, we identify many proteins that are not present in humans, yeasts, or the ancestral alpha-proteobacteria, which can serve as a foundation for future investigations into the unique mitochondria of Dictyostelium. Additionally, we leverage our compendium to highlight the complexity of metabolic reprogramming during starvation-induced development. Our compendium lays a foundation to investigate mitochondrial processes that are unique in protists, as well as for future studies to understand the functions of conserved mitochondrial proteins in health and diseases using $D$. discoideum as the model. 


\section{Introduction}

2 Dictyostelium discoideum, a social amoeba, is a well-established model organism to study 3 eukaryotic cellular processes such as cell motility, chemotaxis, and differentiation (Bozzaro, 2013).

4 Under normal nutrient conditions, $D$. discoideum grows axenically through binary fission (Kessin, 5 2001). However, upon starvation, amoebae secrete cAMP, which attracts neighboring cells to 6 aggregate together and form a multicellular mound. Cells in a mound move collectively as a slug toward light, heat, or humidity to find a suitable environment. The slug eventually matures into a

8 fruiting body consisting of two major types of differentiated cells, spore cells that will start a new life 9 cycle and stalk cells that form a stalk to hold the spore aloft (Kay, 1982). As many of the 10 aforementioned biological processes are intertwined with cellular energetics, investigation of 11 mitochondrial biogenesis and functions is an emerging area in $D$. discoideum research (Francione et 12 al., 2011; Pearce et al., 2019).

14 The $D$. discoideum mitochondrial genome is $\sim 56 \mathrm{~kb}$, circular, double-stranded DNA that encodes two 15 ribosomal RNAs, 18 transfer RNAs (tRNAs), five open reading frames without annotated function, 16 and 38 proteins including 18 subunits of the electron transport chain complexes and 15 ribosomal 17 proteins (Ogawa et al., 2000). Phylogenetic studies reveal that Amoebazoa diverged before 18 Opisthokonta, but after the divergence of Plantae (Baldauf and Doolittle, 1997), and are more closely 19 related to animals than plants. Notably, the Dictyostelium mitochondrial genetic system possesses a 20 few differences from metazoans (Pearce et al., 2019). D. discoideum mitochondrial DNA (mtDNA) 21 has four introns in cox1/2 genes and utilizes universal codons (Angata et al., 1995; Ogawa et al., 22 2000), a common feature of most plants' mitochondria (Jukes and Osawa, 1990; Cho et al., 1998).

23 The universal genetic code and the lack of a full set of tRNA genes on the Dictyostelium 24 mitochondrial genome indicate that some nuclear-encoded tRNAs are likely imported into 25 mitochondria to support the organellar translation. Additionally, the electron transport chain in 26 Dictyostelium contain an additional component compared to its metazoan counterparts: an 27 alternative oxidase (AOX) (Pearce et al., 2019), which is found across eukaryotic clades besides 28 animals (McDonald et al., 2008). AOX is highly expressed during vegetative growth, but its 29 expression level is markedly reduced upon starvation, suggesting a potential metabolic 30 reprogramming occurs during starvation-induced development (Jarmuszkiewicz et al., 2002). 31 Interestingly, either reduction of mtDNA content or disruption of the rps4 locus (encoding mt32 ribosomal protein S4) on mtDNA impairs aggregation and slug phototaxis but has no impact on 33 vegetative growth (Chida, 2004; Chida et al., 2008), suggesting that mtDNA, and most likely an 34 intact oxidative phosphorylation system is essential to initiate the development program. On the 35 other hand, pharmacological inhibitions of either Complex I or Complex $V$ can induce aggregation, 36 even though mitochondrial respiration appears to increase at the beginning of starvation (Kelly et al., 
1 2021). Therefore, the interplay between mitochondrial function and Dictyostelium development 2 remains to be explored.

3 Despite the growing interest in using $D$. discoideum as a model organism to study many conserved 4 mitochondrial processes and some unique biology, a comprehensive list of the mitochondrial 5 proteins has yet to be established. A recent proteomic study detected 294 proteins in $D$. discoideum 6 mitochondria (Mazur et al., 2021), which we believe is far from complete. Nuclear-encoded 7 mitochondrial proteins, which constitute over $90 \%$ of the total mitochondrial proteome, are 8 synthesized in the cytoplasm and then imported to mitochondria. It is estimated that the import of $9 \sim 60 \%$ of these proteins relies on a positively charged, $\mathrm{N}$-terminal mitochondrial targeting sequence 10 (MTS) (Vögtle et al., 2009). Computational approaches that integrate machine learning and known 11 biological data are frequently used to predict mitochondrial targeting based on the presence of an 12 MTS (Almagro Armenteros et al., 2019). However, this method is insufficient to capture all 13 mitochondrial proteins, as most proteins on the outer membrane and in the inner membrane space, 14 and some inner membrane proteins rely on alternate translocation mechanisms. An alternative 15 computational approach leverages sequence homology to known mitochondrial protein 16 compendiums that were generated using mass spectrometry (MS)-based proteomic discovery 17 (Pagliarini et al., 2008; Morgenstern et al., 2017). However, to compensate for the rapid evolution of 18 the mitochondrial genome, nuclear-encoded mitochondrial proteins evolve faster than other nuclear19 encoded proteins (Cole et al., 1995; Sloan et al., 2014; Havird et al., 2015; Li et al., 2017; Yan et al., 20 2019). Thus, some Dictyostelium mitochondrial proteins may escape the homology search, and 21 protist-specific mitochondrial proteins will certainly be missed.

23 In this study, we combined quantitative proteomics and mathematical modeling to identify over 900 24 high-confidence mitochondrial proteins, which were validated through both in silico and fluorescent 25 microscopy analyses. We further complemented the proteomics-based mitochondrial protein 26 discovery with bioinformatic approaches to create a compendium of $1082 \mathrm{D}$. discoideum 27 mitochondrial proteins. We also discuss conserved $D$. discoideum mitochondrial proteins that may 28 be used as the basis of validating mitochondrial proteins in other organisms, as well as unique 29 features of the mitochondrial proteome in $D$. discoideum. 


\section{Results and Discussion}

\section{Mitochondrial protein discovery using quantitative proteomics}

3 To identify putative $D$. discoideum mitochondrial proteins, we searched for Dictyostelium homologs 4 of 1136 human mitochondrial proteins listed in the Human MitoCarta 3.0 (Morgenstern et al., 2017; 5 Rath et al., 2021), and retrieved 616 proteins (Figure 1A, Table S1). This number is much less than 6 known mitochondrial proteins in humans (1136) and baker's yeast (901) (Pagliarini et al., 2008; Rath 7 et al., 2021). We posited that mitochondrial proteome might be highly divergent between $D$. 8 discoideum and humans, and many Dictyostelium mitochondrial proteins might be missed from this 9 bioinformatic curation. We, therefore, took a proteomic approach to directly identify mitochondrial 10 proteins in $D$. discoideum (Figure $1 \mathrm{~A}$ ). From AX2 axenic cultures, we prepared mitochondria 11 isolates—both crude and highly purified—through Percoll gradient ultracentrifugation. We performed 12 tandem mass tag (TMT) liquid chromatography-mass spectrometry (LC-MS) on both mitochondria 13 isolates and included AX2 whole-cell lysate as the control. A total of 6,892 proteins were captured in 14 all samples (Table S2).

16 A limitation of identifying organellar proteins from their subcellular fractions alone is that high17 abundance contaminants are often co-purified and result in false-positive hits. To address this issue, 18 we assessed the probability of a protein localizing to mitochondria by comparing its relative 19 enrichment in mitochondrial preparations to a list of 47 authentic mitochondrial proteins that includes 20 components of electron transport chain complexes and conserved enzymes in citrate cycles (Table 21 S3). We first calculated the ratio of a protein's abundance in the mitochondria isolates, both crude

22 and highly purified, versus its abundance in the whole-cell lysate. The resulting value, indicating its 23 enrichment in mitochondrial preparations, was further normalized to the average enrichment ratio of 24 the 47 reference mitochondrial proteins, to compute the relative enrichment ratio (RER). Overall, a 25 protein's RER in crude mitochondria isolate is in accordance with that in purified mitochondria 26 (Figure 2A). However, the distribution of RERs appears continuous in crude mitochondria (Figure 27 1C), but clusters into two distinct populations in purified mitochondria (Figure 1C), which allowed us 28 to determine a proper threshold of RER for mitochondrial proteins using mathematical modeling. 29 Thus, we proceeded to analyze the RER for purified mitochondria only.

31 In principle, a true mitochondrial protein would be co-purified with the reference mitochondrial 32 proteins in pure mitochondrial isolates, and its RER should be 1.0. However, the RER distribution of 33 these 47 reference proteins (Figure 2B) appears as a normal curve centered around 1.0, suggesting 34 that many mitochondrial proteins may have an RER below 1.0. Among all proteins profiled using 35 TMT-based LC-MS, only 259 have an RER higher than 1.0 in purified mitochondria (Figure 2C). We 36 posit that different mitochondria proteins might be degraded to different extents, based on their 
1 intrinsic stability, during the procedure of mitochondrial purification, which involves overnight

2 ultracentrifugation. Therefore, it is necessary to determine a proper RER value to differentiate

3 mitochondrial proteins from non-mitochondrial proteins. We applied the expectation-maximization

4 (EM) approach to a gaussian mixture model (GMM) to bin all proteins into two clusters: non-

5 mitochondrial and mitochondrial proteins based on their RER values (Figure2D). We chose an RER

6 cutoff of 0.343 (Figure 2D) and assigned a total of 908 proteins having an RER higher than 0.343 as

7 putative mitochondrial proteins (Table S4). GMM predicts that less than $17 \%$ proteins in the

8 mitochondrial cluster, and only $0.1 \%$ proteins in the non-mitochondrial cluster would spill over to the

9 other group (Figure 2D), which corresponds to an $83 \%$ recovery rate and $7 \%$ false discovery rate,

10 respectively.

12 Validation of mitochondrial protein discovery based on quantitative proteomics

13 To validate the accuracy of RER-based mitochondrial protein discovery, we first assessed the 14 recovery rate of putative mitochondrial proteins in silico. Many mitochondrial proteins possess an $\mathrm{N}$ 15 terminus mitochondrial targeting sequence (MTS) that directs the import of nuclear-encoded 16 mitochondrial proteins into the mitochondrial matrix (Backers, 2017). Overall, 24\% of all proteins 17 retrieved in the proteomics discovery experiment contain an MTS (Table S4). Importantly, 94\% of 18 these MTS-bearing proteins had an RER greater than 0.343 (Figure 3A). On the contrary, $96 \%$ of 19 proteins that were destined to other organelles such as the ER, Golgi, lysosomes, vacuoles, or 20 secretory pathway had an RER less than 0.343 . These analyses demonstrate that a cutoff value of 21 an RER at 0.343 effectively separates mitochondrial proteins from non-mitochondrial proteins.

23 We also surveyed the localization of 81 proteins recovered in LC-MS (Table S5), using fluorescent 24 microscopy. These proteins were selected on the basis that their subcellular localization has not 25 been annotated previously as mitochondrial, and their RERs are randomly distributed from 0.1 to 26 1.5. Each protein was tagged with GFP at its C-terminus and co-expressed with an MTS-mCherry 27 fusion protein, which marks mitochondria in D. discoideum AX2 cells. Among the 81 proteins, $90 \%$ of 28 proteins with an RER higher than 0.343 showed complete or partial mitochondrial localization 29 (Figure 3B, 3D), whereas only $5 \%$ of proteins with an RER less than 0.343 showed mitochondrial 30 localization (Figure 3B, 3D), demonstrating a strong positive correlation between RER value and 31 probability of mitochondrial localization (Figure 3B). Moreover, logistic regression analysis on the 32 localization pattern of these 81 proteins predicts that a protein has more than a $78 \%$ probability of 33 localizing to the mitochondria if its RER is higher than 0.343 (Figure $3 \mathrm{C}$ ).

34

35 A comprehensive mitochondrial protein compendium in D. discoideum. 
1 To further improve the coverage and accuracy of the mitochondrial protein discovery, we revised the

2 list based on the in vivo microscopy validation by removing four non-mitochondrial localizing proteins

3 and adding two mitochondrial localizing proteins. We also integrated three sets of mitochondrial

4 protein discovery: the aforementioned list of mitochondrial proteins identified from quantitative

5 proteomics analyses, those retrieved from homology detection, and those retrieved during a gene

6 ontology search for mitochondrial genes. Among the 616 D. discoideum homologs of human

7 mitochondrial proteins (Table S1), 352 proteins have an RER higher than 0.343 and hence were

8 already included in the list, 223 proteins have an RER lower than 0.343 , and 41 proteins were not

9 captured in LC-MS. Among the 264 proteins that were not included in the list, 113 proteins do not

10 have a predicted MTS (Table S1), whereas their human homologs have MTSs, suggesting these

11 proteins might localize to other cellular compartments in $D$. discoideum. An exception is ribosomal

12 protein $\mathrm{S} 14$ (O21035), which is encoded in the nuclear genome in humans but is encoded in the

13 mitochondrial genome in D. discoideum, and thus contains an MTS in human cells but lacks one in

14 D. discoideum. We added those remaining 152 proteins to the list, as well as 32 proteins with

15 mitochondrial gene ontologies that had not emerged during the proteomic or homology analysis. The

16 final compendium consists of 1082 high-confidence mitochondrial proteins in D. discoideum (Table

17 S6).

19 Characterization of the $\boldsymbol{D}$. discoideum mitochondrial proteome

20 Out of the $1082 D$. discoideum mitochondrial proteins, there are 627 and 458 proteins that have

21 homologs in the mitochondrial proteome of human and Saccharomyces cerevisiae, respectively

22 (Figure 4A), indicating that $D$. discoideum mitochondria are more closely related to mitochondria in

23 metazoans than fungi. Only 324 D. discoideum mitochondrial proteins have homologs in Rickettsia

24 prowazekii (Figure 4A), an a-proteobacteria that is closely related to the mitochondrial ancestor.

25 Overall, a total of 313 proteins, representing $28.9 \%$ of the $D$. discoideum mitochondrial proteome,

26 have no homologs in the whole proteome of humans, S. cerevisiae or $R$. prowazekii (Figure 4A,

27 Table S6), indicating that a large fraction of $D$. discoideum mitochondrial proteins was evolved de

28 novo after the divergence of Amoebozoa. Moreover, 75 D. discoideum mitochondrial proteins (6.9\%)

29 have no homologs in $D$. purpureum (Figure 4A), a closely related species of social amoeba, further

30 substantiating the fast-evolving nature of the amoeba mitochondrial proteome.

32 There are $89 D$. discoideum mitochondrial proteins (8.2\%) with human homologs that had not been

33 annotated as mitochondrial proteins (Figure 4B, Table S6). Among these 89 proteins, 74 were also

34 not annotated as mitochondrial proteins in yeast, including 32 that had homologs in $S$. cerevisiae.

35 Given the estimated false-discovery rate of our compendium, the localization of these proteins needs

36 to be experimentally accessed. Nonetheless, there are a few examples, such as the RNB domain- 
1 containing protein (DDB_G0288469) and tRNA-binding domain-containing protein

2 (DDB_G0349377), both of which have predicted MTSs and are likely targeted to the mitochondrial

3 matrix. The human homologs of DDB_G0288469, DIS3-like exonuclease 2, and DDB_G0349377,

4 rhomboid-related protein 4, were not included in the human compendium (Rath et al., 2021), despite

5 evidence that the yeast homolog of DIS3-like exonuclease 2 localizes to the mitochondria (Pagliarini

6 et al., 2008), and that rhomboid-related protein 4 has been partially shown to localize to the

7 mitochondria. The mitochondrial localization of their $D$. discoideum homologs substantiates these

8 two proteins might indeed localize to the mitochondria and indicates that our compendium can

9 complement previous studies toward a more comprehensive discovery of mitochondrial proteins in

10 other organisms.

12 Additionally, we categorized the $D$. discoideum mitochondrial proteome using PANTHER biological

13 function or protein family classifications (Figure 4B, Table S6). Proteins involved in mitochondrial

14 gene expression and metabolism comprise the largest fractions of all mitochondrial proteins, over

$1520 \%$ for each category. Other proteins are involved in mitochondrial protein homeostasis, the 16 electron transport chain, redox signaling and metabolism, and regulation of mitochondrial

17 morphology and dynamics. A large fraction of $D$. discoideum mitochondrial proteins, approximately

$1815 \%$, have no classified functions based on PANTHER analyses (Figure 4B).

\section{D. discoideum-specific mitochondrial proteins}

21 Proteins involved in gene expression consisted of a large fraction of $D$. discoideum specific

22 mitochondrial proteome (Figure 4B), reflecting that the $D$. discoideum mitochondrial genome is more

23 complex than human mtDNA. On the contrary, few metabolism proteins emerged in the list (Figure

24 4B), suggesting that metabolic processes are highly conserved between $D$. discoideum and

25 metazoans. Here, we expand upon a few of the unique features of the $D$. discoideum mitochondrial

26 protein compendium.

28 Mosaic nature of mitochondrial ribosomes

29 Mitochondrial ribosomes (mitoribosomes), ribosomal assembly factors, and other proteins involved

30 in translation represented $8.9 \%$ and $9.3 \%$ of the overall and unique mitochondrial protein

31 compendium, respectively. While mitoribosomes are thought to be evolved from bacterial ribosomes,

32 these two differ greatly with regards to their structure, function, as well as their composition of

33 proteins and RNAs. We identified 51 proteins that are predicted to be mitoribosomal proteins,

34 including 13 proteins that did not share significant homology with any $H$. sapiens, $S$. cerevisiae, or $R$.

35 prowazekii proteins (Table S7). Interestingly, D. discoideum mitoribosomal proteins belong to

36 families across several taxonomic groups: 35 proteins belong to mammalian mitoribosomal protein 
1 families (28s and 39s), 2 belong to eukaryotic cytosolic ribosomal protein families (60s), 9 belong to

2 yeast mitoribosomal protein families ( $37 \mathrm{~s}$ and 54s), 2 belong to chloroplast or bacterial ribosomal

3 protein families (30s and 50s), 1 is from archaea, and 2 are universally conserved among

4 prokaryotes and eukaryotes. It has previously been shown that cytosolic ribosomes tether to the

5 mitochondrial outer membrane. Hence, the recovery of the $60 S$ ribosomal protein L22 could be the

6 result of the association of cytoplasmic ribosome with the mitochondrial outer membrane rather than

7 its localization in the matrix (Gold et al., 2017). Nonetheless, the presence of proteins representing

8 multiple mitoribosome lineages suggests that there may be $D$. discoideum or protist-specific

9 mechanisms to process mitochondrial transcripts and to regulate mitochondrial translation. Further

10 validation of these findings is necessary as the composition and structure of the $D$. discoideum

11 mitoribosome have yet to be resolved.

13 Mitochondrial DNA and RNA processing factors

14 Among the list of unique proteins are 24 candidate mtDNA and mtRNA processing factors including

15 five endonucleases and a pentatricopeptide repeat (PPR)-containing protein A (PtcA). Bioinformatic

16 analysis suggests that PtcA belongs to the mitochondrial group I intron splicing family. PPR proteins,

17 defined by tandem PPR domains, are implicated in several different mitochondrial gene expression

18 processes including translation initiation, and ribosomal stabilization (Manna, 2015). The number of

19 PPR proteins that are encoded in an organism varies greatly: terrestrial plants, such as Arabidopsis

20 thaliana, have upwards of 450 PPR proteins, while humans have 7 (Lurin et al., 2004; Lightowlers

21 and Chrzanowska-Lightowlers, 2013). D. discoideum has 12 PPR-domain containing peptides,

22 including PtcA, reflecting a greater complexity of $D$. discoideum's mitochondrial genome compared

23 to that of metazoans (Manna et al., 2013).

25 Divergent evolution path of lipid biosynthesis

26 The mevalonate pathway, which produces five-carbon blocks for the synthesis of diverse

27 biomolecules such as cholesterol and coenzyme Q10, is an essential and highly conserved process

28 in eukaryotes, archaea, and some bacteria. In animals and fungi, the mevalonate pathway takes

29 place in ER, and 3-hydroxy-3-methylglutaryl (HMG)-coenzyme A (CoA) reductase (HMGR), a key

30 enzyme in this pathway that converts HMG-CoA to mevalonate, localizes in the ER and peroxisomes

31 (Chin et al., 1984; Keller et al., 1986; Burg and Espenshade, 2011). HMGR2, one of two HMG

32 reductases in $D$. discoideum, is recovered in our compendium and contains a predicted MTS,

33 suggesting that it likely localizes to the mitochondrial matrix. Additionally, HGSA, one of the two

34 HMG-CoA synthases, also emerged as a mitochondrial protein. Our mitochondrial protein discovery

35 suggests that mevalonate metabolism may take place in mitochondria in $D$. discoideum, highlighting 
1 the evolutionary divergence of some metabolic pathways that originated from the common

2 mitochondrial ancestor.

4 Implication of mitochondrial function in multicellular development

5 D. discoideum with reduced mtDNA or a disruption of the gene encoding mt-ribosomal protein S4 6 display no defect in vegetative growth but have impaired starvation-induced development, 7 suggesting that mitochondrial respiration is necessary for multicellularity (Chida, 2004; Chida et al., 8 2008). However, contrasting evidence has demonstrated a significant decrease in mitochondrial 9 respiration after respiration, and accordingly, a decreasing expression of many respiration 10 complexes (Kelly et al., 2021). To understand potential regulations of mitochondrial function in 11 multicellular development, we retrieved RNA sequencing data using the Dictyostelium gene 12 expression database, dictyExpress (Parikh et al., 2010; Stajdohar et al., 2017).

14 Overall, there was a decrease in the expression of mitochondrial genes within our compendium over 15 the 24-hr development time course (Figure 5A). A similar pattern is observed in proteins that are 16 involved in mitochondrial DNA maintenance and gene expression. Interestingly, despite the 17 decrease in gene expression machinery (Figure 5B), over half of the mitochondria-encoded genes in 18 the dataset (19 of 35 ) were upregulated ( $\log 2 \mathrm{FC} \geq 1$ ) after starvation induction (Figure $5 \mathrm{C}$ ). Further, 19 in examining all respiratory chain complexes, 12 nuclear-encoded ETC subunits had a higher 20 expression level $(\log 2 \mathrm{FC} \geq 1)$ at or after 12 hours of starvation (Figure 5D), besides the 10 nuclear or 21 mitochondrial-encoded subunits that show a burst of expression in the first 4 hours after the 22 starvation (Figure 5D). The complex pattern of mitochondrial gene expression, particularly the 23 upregulation of electron transport chain complex subunits during the development suggests potential 24 roles of mitochondrial respiration in Dictyostelium development, and that both nuclear and 25 mitochondrial-encoded proteins are likely implicated in these processes.

\section{Conclusion}

28 Here, we generated the most comprehensive list of mitochondrial proteins in $D$. discoideum to date.

29 Our compendium lays the foundation for future studies to understand the functions of conserved 30 mitochondrial proteins in health and diseases using $D$. discoideum as the model. It also provides an 31 entry to study many fascinating mitochondrial processes that are unique in protists. Additionally, 32 thorough comparative genomics, our compendium will complement mitochondrial protein discovery 33 in other organisms and may shed light on the evolution of mitochondrial proteome and processes. 


\section{Materials and Methods:}

\section{Cell culture and transformation}

3 Dictyostelium discoideum AX2 cultures were maintained in HL5 medium at $22{ }^{\circ} \mathrm{C}$ (Fey et al., 2007).

4 Transformants were generated via electroporation as previously described (Gaudet et al., 2007),

5 with modifications. After electroporation (BioRad Genepulser), cells were incubated on ice for 10 6 minutes. Subsequently, cells were transferred from the cuvette with $2 \mathrm{~mL}$ of HL5 and plated onto 12-

7 well tissue culture plates. After 24 hours, transformants were selected with Genectin and/or

8 Blasticidin S (Thermo Fisher, $10 \mu \mathrm{g} / \mathrm{mL}$ each) in HL5.

10 Protein mass spectrometry

11 Mitochondrial isolation

12 Cells were harvested at a concentration of $1-3 \times 10^{6}$ cells $/ \mathrm{mL}$ and resuspended at $2 \times 10^{7}$ cells $/ \mathrm{mL}$ in $13800 \mu \mathrm{L}$ of Reagent A from the Mitochondrial Isolation Kit for Cultured Cells (Thermo Fisher \#89874) 14 on ice. Cell lysis and crude mitochondrial preparation were performed as previously described with 15 modifications (Graham, 1999; Glancy and Balaban, 2011). Cells were lysed with 35 strokes of a 16 Dounce homogenizer followed by the addition of an equal volume of Reagent $\mathrm{C}$. Whole-cell lysate 17 samples were stored at $-80^{\circ} \mathrm{C}$ or were centrifuged three times $\left(700 \times \mathrm{g}, 10\right.$ minutes, $\left.4{ }^{\circ} \mathrm{C}\right)$ to purify 18 the mitochondria. For each centrifugation step, the supernatant was transferred to a fresh $1.5 \mathrm{~mL}$ 19 tube. The crude mitochondrial lysate was used immediately for purification or was stored at $-80^{\circ} \mathrm{C}$.

21 To generate purified mitochondrial isolates, Percoll gradient centrifugation was performed as follows.

22 Lysis suspension (1-2 mL) was added to the top of a Percoll (Cytiva) and Development Buffer (DB)

$23\left(5 \mathrm{mM} \mathrm{Na}_{2} \mathrm{HPO}_{4}, 5 \mathrm{mM} \mathrm{KH}_{2} \mathrm{PO}_{4}, 1 \mathrm{mM} \mathrm{CaCl}\right.$, $2 \mathrm{mM} \mathrm{MgCl}$, $\mathrm{pH}=6.5$ ) solution (8 mL, 30\% Percoll)

24 in a $10 \mathrm{~mL}$ ultracentrifuge tube. Ultracentrifugation $(68,000 \times \mathrm{g}, 40$ minutes) yielded three distinct

25 layers. The top layer, containing contaminants, was discarded. The middle layer, containing 26 mitochondria, was transferred into a fresh $2 \mathrm{~mL}$ tube. Aliquots of the mitochondrial suspension were 27 topped off with $500 \mu \mathrm{L}$ of $\mathrm{DB}$, then centrifuged $\left(13,000 \times \mathrm{g}, 10\right.$ minutes, $\left.4{ }^{\circ} \mathrm{C}\right)$. Following 28 centrifugation, the supernatant was aspirated, and the mitochondria-containing pellet was 29 maintained on ice. To lyse the mitochondria, the pellet was washed with $2 \mathrm{~mL}$ of $\mathrm{DB}$, centrifuged $30\left(10,000 \times \mathrm{g}, 10\right.$ minutes, $\left.4^{\circ} \mathrm{C}\right)$, and resuspended in $2 \mathrm{~mL}$ of $\mathrm{DB}$ with urea $(8 \mathrm{M})$. Protein yield was 31 quantified via Bradford Assay (BioRad) according to the manufacturer's protocol.

33 Relative protein quantification

34 Resuspended cell pellets were lysed via pulsed sonication, then sequentially reduced, alkylated, and 35 digested overnight with trypsin. The protein digests were labeled with 10-plex Tandem Mass Tag 36 (TMT) reagents (Thermo Fisher Scientific) (Dayon et al., 2008), then were pooled and desalted. To 
separate the peptide mixtures into 24 fractions, high $\mathrm{pH}$ reversed-phase liquid chromatography was performed (Yang et al., 2012). Each fraction was analyzed on an Orbitrap Lumos (Thermo Fisher Scientific) nanoLCMS system.

Peptide and proteins were identified as described in He et al., (2020). In brief, the resulting LCMS raw data were searched against a database downloaded from dictybase.org using the Sequest HT algorithm on the Proteome Discoverer 2.4 platform (Thermo Fisher Scientific). Three groups of samples were normalized to 47 reference mitochondrial proteins.

10 The relative enrichment ( $R E)$ was defined as the ratio of a protein's enrichment from a crude or 11 purified mitochondria sample over its enrichment from a whole-cell lysate sample. To calculate the 12 relative enrichment ratio (RER), REs were normalized such that the average RER of 47 known 13 mitochondrial (TCA cycle, ETC, or OXPHOS) proteins is 1 (Table S3). The RER presented is a 14 median value of three biological replicates.

\section{Mathematical modeling}

17 To classify proteins as mitochondrial or non-mitochondrial based on their RER, the RER distribution

18 of isolated proteins was fit to a Gaussian mixture model (GMM) using the Expectation-Maximization

19 (EM) algorithm in $\mathrm{R}$ (Benaglia et al., 2009). The cutoff value (RER = 0.343) was four times the

20 standard deviation plus the mean of curve 1 (representing non-mitochondrial proteins), such that $99.9 \%$ of the proteins below the cutoff were contained within curve 1 .

\section{Bioinformatic analyses}

24 Homology analyses

25 Protein sequence homology was established by BlastP expect $<0.001$ and bit-score $>40$ (Pearson, 26 2013), or by HMMER sequence e-value $<0.01$. Subcellular localization was predicted using 27 TargetP-2.0 (Almagro Armenteros et al., 2019). Biological functions for all proteins in the $D$. 28 discoideum and human mitochondrial proteome were manually categorized from biological function 29 or protein family classifications provided from PANTHER (The Gene Ontology Consortium et al., 30 2021).

32 In silico dataset correction

33 Two strategies were implemented to supplement the mitochondrial protein discovery. The top $D$. 34 discoideum homolog of human mitochondrial proteins (Table S1, Rath et al., 2021) were curated. 35 Additionally, D. discoideum proteins annotated with the gene ontology term "mitochond" on AmiGO 36 were selected (Carbon et al., 2009). Proteins within these lists were integrated into the final 
1 mitochondrial compendium so long as they had a predicted mitochondrial targeting sequence if their

2 human homolog also had a predicted mitochondrial targeting sequence, except in cases where the

3 D. discoideum protein was mitochondrial-encoded.

RNA sequencing data visualization

6 Normalized RNA-seq data from Parikh et al. (2010) was retrieved using dictyExpress (Stajdohar et 7 al., 2017). For the 1082 proteins in the mitochondrial compendium, only 1075 corresponding genes

8 were present in the dataset. To compare the gene profiles, data were scaled to a mean of 0 and

9 standard deviation of 1 using the scale function in R. For the overall mitochondrial expression profile, 10 genes and timepoints were ordered using hierarchical clustering (heatmaps.2). For the profiles of 11 individual biological processes, scaled data (Table S8) were imported into the matrix visualization

12 software Morpheus (https://software.broadinstitute.org/morpheus) and ordered via hierarchical 13 clustering with one minus Pearson's correlation as the distance metric and average as the linkage 14 method. Gene upregulation was determined by a log2 fold-change $\geq 1$.

\section{Library generation for imaging verification}

17 To evaluate the efficacy of our mitochondrial protein identification, 98 proteins were selected to be 18 GFP tagged so that their localization could be assessed via fluorescence microscopy (Table S5).

19 None of the proteins selected had a gene ontology annotation that indicated mitochondrial 20 localization. Four proteins selected for verification had homologs listed in the human mitochondrial 21 proteome.

23 All genes were synthesized by Gene Universal. Of the 98 genes submitted for synthesis, 85 were 24 generated as inserts in pDM323, a $D$. discoideum extrachromosomal expression vector with G418 25 resistance and a C-terminal GFP tag (Veltman et al., 2009); 6 genes were generated as inserts in 26 the shuttle vector puC57 and were subsequently cloned into pDM323 between Bglll and Spel sites 27 using the In-Fusion® HD Cloning Kit (Takara Bio USA) and confirmed by sequencing. The other 7 28 genes were unable to be synthesized, such that only 92 proteins were screened. Of these 92 genes, 29 only 81 were successfully expressed in $D$. discoideum. The RER of the proteins that were verified 30 were as follows: 14 proteins with a $\mathrm{RER}>.75,11$ proteins with a $\mathrm{RER}=0.75-0.5,24$ proteins with a 31 RER $=.5-0.25$, and 32 proteins with RER $<0.25$. To observe mitochondrial localization, the 32 mitochondrial targeting sequence of respiratory cytochrome oxidase c subunit IV fused with mCherry 33 (CoxIV-mCherry) was cloned into pDM326, a D. discoideum extrachromosomal expression vector 34 with Blasticidin S resistance (Veltman et al., 2009). All primers for cloning are listed in Table S9. 
1 To image cells in the axenic phase, cells $(200 \mu \mathrm{l})$ were transferred to 8 -well glass chambers 7 to 10

2 days post-transformation. Cells were allowed to adhere to the bottom of the chamber for 30 minutes

3 before the media was aspirated. The media was replaced with 1x PBS after three washes (200 $\mu \mathrm{l}$ for

4 all). Confocal images were collected on a PerkinElmer Ultraview system (Zeiss Plan-apochromat

5 63x/1.4 oil lens, Volocity acquisition software, Hamamatsu Digital Camera C10600 ORCA-R2,

6 Immersol immersion oil). Images $(0.5 \mu \mathrm{m}$ z-step) were analyzed with ImageJ (National Institutes of

7 Health) and formatted in Adobe Photoshop.

9 Code and data availability

10 Proteomics data are deposited at ProteomXchange (PXD029101). R code is available from Anna

11 Freitas's GitHub repo https://github.com/freitasav/DD-mitoproteome.

\section{Quantification and Statistical Analyses}

13 All data were presented as the mean \pm SD unless otherwise indicated. $P$ values were calculated in $R$

14 using a one-way analysis of variance (ANOVA) followed by Tukey's post-hoc test to test for the

15 effect of RER on mitochondrial localization. Statistical significance of difference was considered

16 when $\mathrm{p}<0.05$.

18 To predict the probability of localization based on RER, outliers were identified and removed from

19 the microscopy validation dataset based on the interquartile method (median + 1.5 SD). Data were

20 analyzed using a binomial logistic regression ( $\mathrm{glm}$ function in $\mathrm{R}$ ) with excluded from the mitochondria

21 as the reference level, and partial mitochondrial localization, mitochondrial localization, or combined

22 (in which the partial and mitochondrial outcomes are collapsed) as the outcome levels. Predicted

23 probabilities and $95 \%$ confidence intervals were calculated (predict function in $\mathrm{R}$ ) to compare

24 outcomes.

\section{ACKNOWLEDGEMENTS}

27 We thank Dr. Edward Korn for his advice and reagents on D. discoideum culturing; Dr. Raúl Covian

28 Garcia for his advice on mitochondria purification; and dictyBase for various plasmids. This work was

29 supported by the Intramural Research Program of National Heart, Lung, and Blood Institute.

31 DECLARATION OF INTERESTS

32 The authors declare no competing interests.

\section{SUPPLEMENTAL INFORMATION}

35 Supplemental Information includes 9 supplemental tables. 


\section{References}

Almagro Armenteros, JJ, Salvatore, M, Emanuelsson, O, Winther, O, von Heijne, G, Elofsson, A, and Nielsen, H (2019). Detecting sequence signals in targeting peptides using deep learning. Life Sci Alliance 2, e201900429.

Angata, K, Kuroe, K, Yanagisawa, K, and Tanaka, Y (1995). Codon usage, genetic code and phylogeny of

Benaglia, T, Chauveau, D, Hunter, DR, and Young, D (2009). mixtools: An R Package for Analyzing Finite Mixture Models. J Stat Soft 32.

Bozzaro, S (2013). The Model Organism Dictyostelium discoideum. In: Dictyostelium Discoideum Protocols, ed. L Eichinger, and F Rivero, Totowa, NJ: Humana Press, 17-37.

10 Burg, JS, and Espenshade, PJ (2011). Regulation of HMG-CoA reductase in mammals and yeast. Progress in 11 Lipid Research 50, 403-410.

12 Carbon, S, Ireland, A, Mungall, CJ, Shu, S, Marshall, B, Lewis, S, the AmiGO Hub, and the Web Presence

13 Working Group (2009). AmiGO: online access to ontology and annotation data. Bioinformatics 25, 288-289.

14 Chida, J (2004). The necessity of mitochondrial genome DNA for normal development of Dictyostelium cells. 15 Journal of Cell Science 117, 3141-3152.

16 Chida, J, Amagai, A, Tanaka, M, and Maeda, Y (2008). Establishment of a new method for precisely determining the functions of individual mitochondrial genes, using Dictyostelium cells. BMC Genet 9, 25.

18 Chin, DJ, Gil, G, Russell, DW, Liscum, L, Luskey, KL, Basu, SK, Okayama, H, Berg, P, Goldstein, JL, and Brown, MS (1984). Nucleotide sequence of 3-hydroxy-3-methyl-glutaryl coenzyme A reductase, a glycoprotein of endoplasmic reticulum. Nature 308, 613-617.

21 Cho, Y, Qiu, Y-L, Kuhlman, P, and Palmer, JD (1998). Explosive invasion of plant mitochondria by a group I 22 intron. Proceedings of the National Academy of Sciences 95, 14244-14249.

23 Cole, RA, Slade, MB, and Williams, KL (1995). Dictyostelium discoideum mitochondrial DNA encodes a NADH:Ubiquinone oxidoreductase subunit which is nuclear encoded in other eukaryotes. J Mol Evol 40, 616621. Chem 80, 2921-2931.

Fey, P, Kowal, AS, Gaudet, P, Pilcher, KE, and Chisholm, RL (2007). Protocols for growth and development of Dictyostelium discoideum. Nat Protoc 2, 1307-1316.

31 Francione, LM, Annesley, SJ, Carilla-Latorre, S, Escalante, R, and Fisher, PR (2011). The Dictyostelium model 32 for mitochondrial disease. Seminars in Cell \& Developmental Biology 22, 120-130.

33 Gaudet, P, Pilcher, KE, Fey, P, and Chisholm, RL (2007). Transformation of Dictyostelium discoideum with 34 plasmid DNA. Nat Protoc 2, 1317-1324.

35 Glancy, B, and Balaban, RS (2011). Protein composition and function of red and white skeletal muscle 36 mitochondria. 300, 11. 
1 Gold, VA, Chroscicki, P, Bragoszewski, P, and Chacinska, A (2017). Visualization of cytosolic ribosomes on the

2 surface of mitochondria by electron cryo-tomography. EMBO Rep 18, 1786-1800.

3 Graham, JM (1999). Purification of a Crude Mitochondrial Fraction by Density-Gradient Centrifugation.

4 Current Protocols in Cell Biology 4.

5 Havird, JC, Whitehill, NS, Snow, CD, and Sloan, DB (2015). Conservative and compensatory evolution in 6 oxidative phosphorylation complexes of angiosperms with highly divergent rates of mitochondrial genome 7 evolution: MITONUCLEAR COEVOLUTION IN SILENE OXPHOS COMPLEXES. Evolution 69, 3069-3081.

$8 \mathrm{He}, \mathrm{Y}, \mathrm{Chen}, \mathrm{Y}, \mathrm{Morris}, \mathrm{DL}$, Lee, D-Y, and Tjandra, N (2020). Bax expression is optimal at low oxygen tension 9 and constant agitation. Protein Expression and Purification 165, 105501.

10 Jarmuszkiewicz, W, Behrendt, M, Navet, R, and Sluse, FE (2002). Uncoupling protein and alternative oxidase 11 of Dictyostelium discoideum[: occurrence, properties and protein expression during vegetative life and 12 starvation-induced early development. FEBS Letters 532, 459-464.

13 Jukes, TH, and Osawa, S (1990). The genetic code in mitochondria and chloroplasts. Experientia 46, 1117141126.

15 Kay, RR (1982). cAMP and spore differentiation in Dictyostelium discoideum. Proceedings of the National 16 Academy of Sciences 79, 3228-3231.

17 Keller, GA, Pazirandeh, M, and Krisans, S (1986). 3-Hydroxy-3-methylglutaryl coenzyme A reductase

18 localization in rat liver peroxisomes and microsomes of control and cholestyramine-treated animals:

19 quantitative biochemical and immunoelectron microscopical analyses. Journal of Cell Biology 103, 875-886.

20 Kelly, B et al. (2021). Sulfur sequestration promotes multicellularity during nutrient limitation. Nature 591, $21 \quad 471-476$.

22 Kessin, RH (2001). Dictyostelium: Evolution, Cell Biology, and the Development of Multicellularity, Cambridge: 23 Cambridge University Press.

24 Li, Y, Zhang, R, Liu, S, Donath, A, Peters, RS, Ware, J, Misof, B, Niehuis, O, Pfrender, ME, and Zhou, X (2017). 25 The molecular evolutionary dynamics of oxidative phosphorylation (OXPHOS) genes in Hymenoptera. BMC 26 Evol Biol 17, 269.

27 Lightowlers, RN, and Chrzanowska-Lightowlers, ZM (2013). Human pentatricopeptide proteins: Only a few 28 and what do they do? RNA Biology 10, 1433-1438.

29 Lurin, C et al. (2004). Genome-Wide Analysis of Arabidopsis Pentatricopeptide Repeat Proteins Reveals Their 30 Essential Role in Organelle Biogenesis[W]. The Plant Cell 16, 2089-2103.

31 Manna, S (2015). An overview of pentatricopeptide repeat proteins and their applications. Biochimie 113, 32 93-99.

33 Manna, S, Brewster, J, and Barth, C (2013). Identification of Pentatricopeptide Repeat Proteins in the Model 34 Organism Dictyostelium discoideum. International Journal of Genomics 2013, 1-8. 
1 Mazur, M, Wojciechowska, D, Sitkiewicz, E, Malinowska, A, Świderska, B, Kmita, H, and Wojtkowska, M

2 (2021). Mitochondrial Processes during Early Development of Dictyostelium discoideum: From Bioenergetic

3 to Proteomic Studies. Genes 12, 638.

4 Morgenstern, M et al. (2017). Definition of a High-Confidence Mitochondrial Proteome at Quantitative Scale.

5 Cell Reports 19, 2836-2852.

6 Ogawa, S et al. (2000). The mitochondrial DNA of Dictyostelium discoideum: complete sequence, gene

7 content and genome organization. Mol Gen Genet 263, 514-519.

8 Pagliarini, DJ et al. (2008). A Mitochondrial Protein Compendium Elucidates Complex I Disease Biology. Cell

9 134, 112-123.

10 Parikh, A et al. (2010). Conserved developmental transcriptomes in evolutionarily divergent species. Genome 11 Biol 11, R35.

12 Pearce, XG, Annesley, SJ, and Fisher, PR (2019). The Dictyostelium model for mitochondrial biology and 13 disease. Int J Dev Biol 63, 497-508.

14 Pearson, WR (2013). An Introduction to Sequence Similarity ("Homology") Searching. Current Protocols in 15 Bioinformatics 42.

16 Rath, S et al. (2021). MitoCarta3.0: an updated mitochondrial proteome now with sub-organelle localization 17 and pathway annotations. Nucleic Acids Research 49, D1541-D1547.

18 Sloan, DB, Triant, DA, Wu, M, and Taylor, DR (2014). Cytonuclear Interactions and Relaxed Selection 19 Accelerate Sequence Evolution in Organelle Ribosomes. Molecular Biology and Evolution 31, 673-682.

20 Stajdohar, M, Rosengarten, RD, Kokosar, J, Jeran, L, Blenkus, D, Shaulsky, G, and Zupan, B (2017). dictyExpress: a web-based platform for sequence data management and analytics in Dictyostelium and

22 beyond. BMC Bioinformatics 18, 291.

23 The Gene Ontology Consortium et al. (2021). The Gene Ontology resource: enriching a GOld mine. Nucleic 24 Acids Research 49, D325-D334.

25 Veltman, DM, Akar, G, Bosgraaf, L, and Van Haastert, PJM (2009). A new set of small, extrachromosomal 26 expression vectors for Dictyostelium discoideum. Plasmid 61, 110-118.

27 Vögtle, F-N et al. (2009). Global Analysis of the Mitochondrial N-Proteome Identifies a Processing Peptidase 28 Critical for Protein Stability. Cell 139, 428-439.

29 Yan, Z, Ye, G, and Werren, JH (2019). Evolutionary Rate Correlation between Mitochondrial-Encoded and 30 Mitochondria-Associated Nuclear-Encoded Proteins in Insects. Molecular Biology and Evolution 36, 1022$31 \quad 1036$.

32 Yang, F, Shen, Y, Camp, DG, and Smith, RD (2012). High-pH reversed-phase chromatography with fraction 33 concatenation for 2D proteomic analysis. Expert Review of Proteomics 9, 129-134. 
Figures and Figure Legends
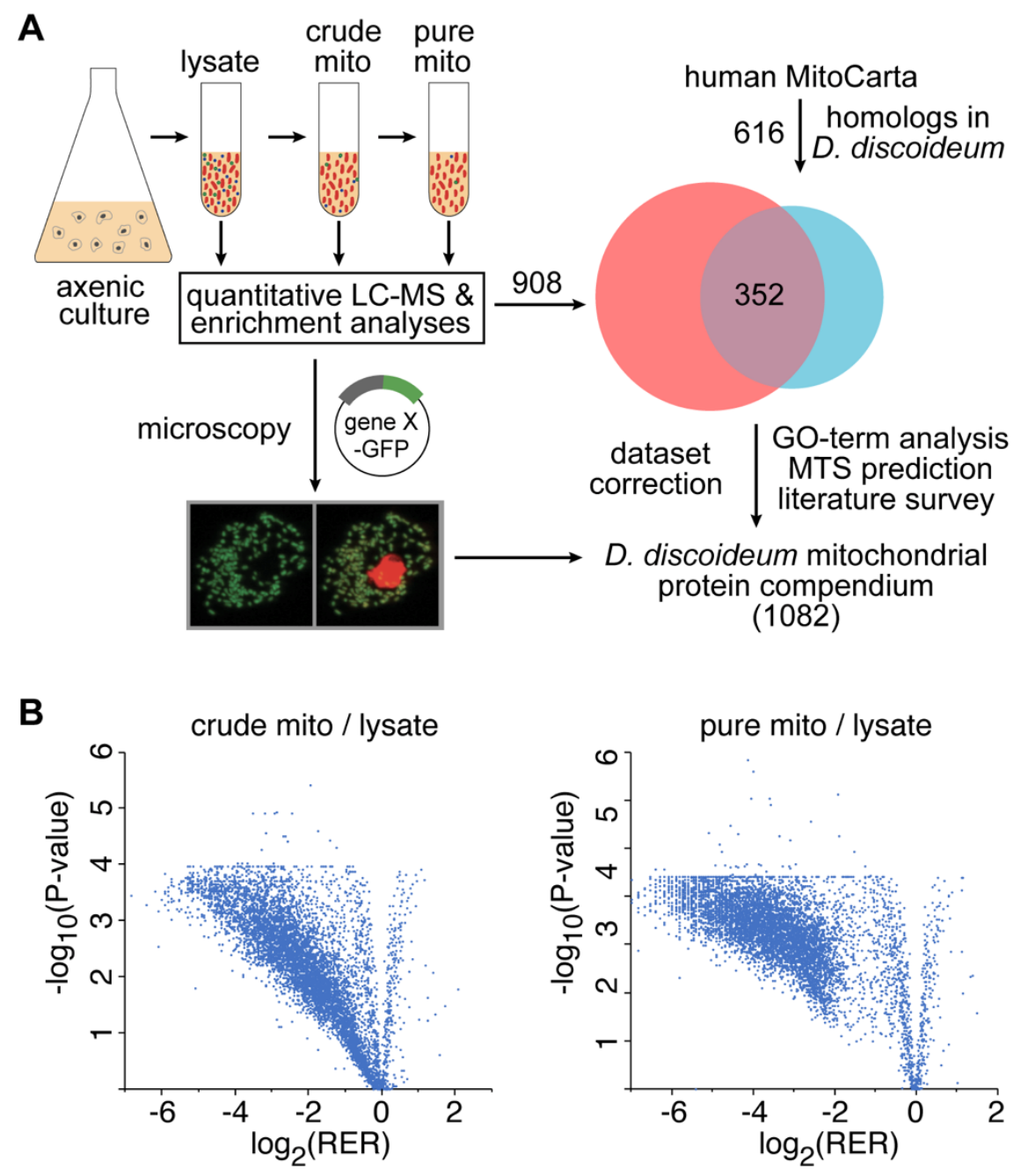

Figure 1. Curation of a comprehensive mitochondrial proteome in Dictyostelium discoideum. (A) A homology search against human mitochondrial protein sequences yielded a list of 616 putative $D$. discoideum mitochondrial proteins. To identify additional mitochondrial proteins, we performed quantitative MS for proteins identified in the whole-cell lysate, as well as in crude and purified mitochondrial samples. Microscopy was used to validate MS and enrichment analyses. After correcting the proteomic dataset based on microscopy results, we incorporated the homology and proteomic analyses to yield a comprehensive mitochondrial protein compendium. (B) Volcano plots displaying the -log10 ( $p$-value) versus log2 (relative enrichment ratios) of proteins in crude mitochondrial or pure mitochondrial versus whole-cell lysate samples. Data are presented as means $(n=3)$. 
A

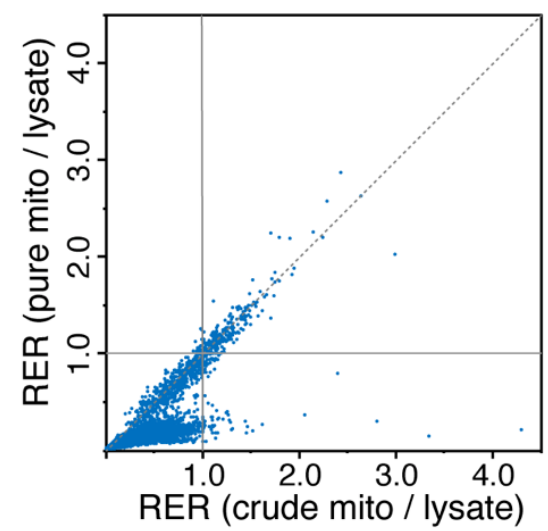

C

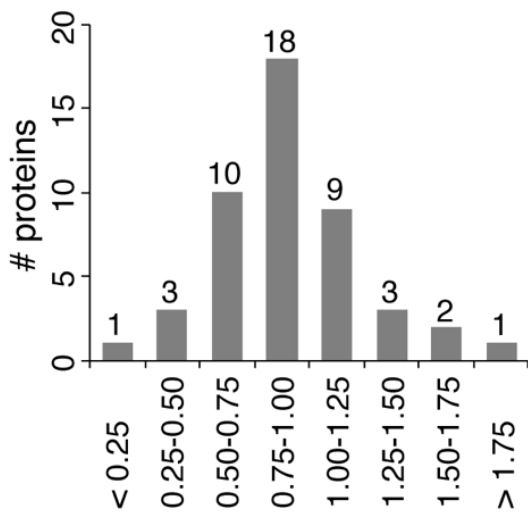

RER (pure mito / lysate)
B

\begin{tabular}{lc} 
RER & \# proteins \\
\hline$>1.50$ & 25 \\
$1.00-1.50$ & 234 \\
$0.75-1.00$ & 277 \\
$0.50-0.75$ & 241 \\
$0.25-0.50$ & 346 \\
$<0.25$ & 5769 \\
total & 6892 \\
\hline
\end{tabular}

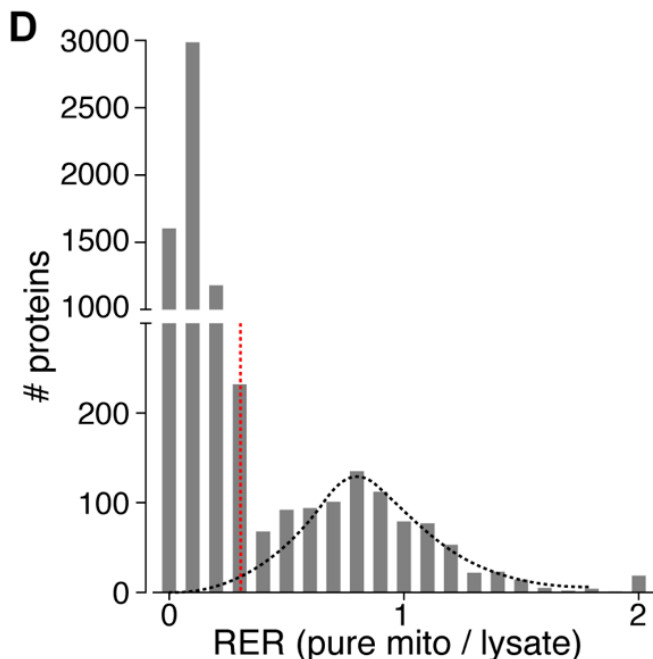

Figure 2. Prediction of mitochondrial localization based on relative enrichment analysis. (A) Crude mitochondrial RER versus pure mitochondrial RER for all samples quantified in the proteomic discovery experiment. The dashed line represents equal crude and pure RERs. Data are presented as means $(n=3)$. (B) Summary table of pure mitochondrial relative enrichment ratios for all proteins in the protein discovery experiment. (C) Distribution of core D. discoideum mitochondrial ETC, OXPHOS, and TCA cycle proteins $(n=47)$ based on RER. (D) The distribution of mitochondrial $(n=$ 9 908) and non-mitochondrial proteins $(n=5984)$ based on RER follow a Gaussian mixture model, in which a cutoff value (red, RER $=0.343$ ) separates two normal curves. 
A

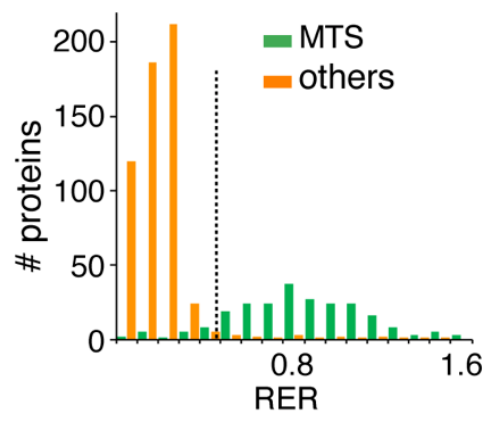

B

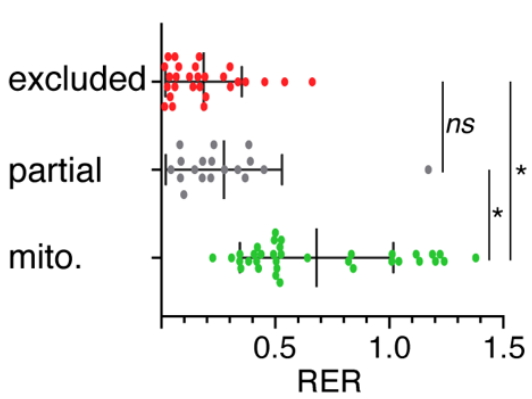

C

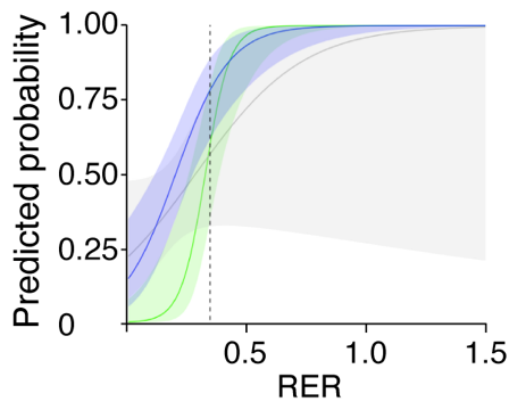

D mito.

partial

ER

puncta

cytoplasm

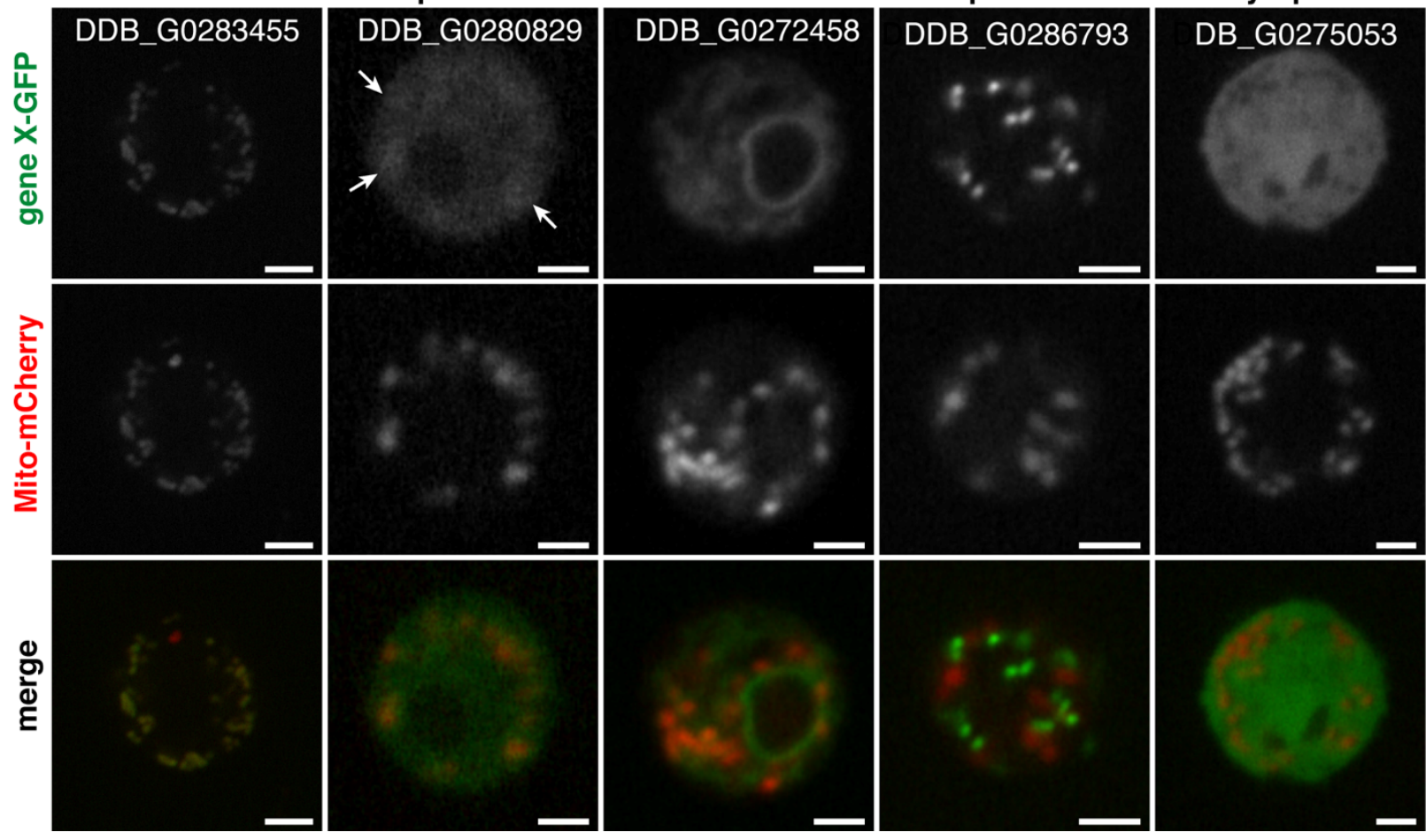

Figure 3. Validation of proteomic discovery and enrichment analyses. (A) Distribution of all proteins quantified with a predicted mitochondrial targeting sequence (MTS) or signal peptide sequence. The dashed line represents the RER cutoff for predicting mitochondrial localization (0.343). (B) Dot plot with interquartile ranges of proteins that had mitochondrial localization (green), partial mitochondrial localization (gray), or were excluded from the mitochondria (red). ${ }^{*}, p \leq 5 \times 10^{-5}$. (C) Predicted probabilities and confidence intervals as analyzed by logistic regression for mitochondrial (green), partial mitochondrial (gray), and either mitochondrial or partial (blue) localization. The dashed line represents the RER cutoff for predicting mitochondrial localization (0.343).

(D) Representative live-cell confocal images of axenic stage

D. discoideum expressing GFP-tagged genes of interest leveled with cytochrome oxidase c subunit IV tagged with mCherry (Mito-mCherry). Arrowheads denote areas of partial localization.

14 
A

D. discoideum

(1082)

\begin{tabular}{lr}
\hline H. sapiens (1136) & 627 \\
S. cerevisiae (901) & 458 \\
R. prowazekii (832) & 324 \\
D. purpureum (?) & 1007 \\
\hline
\end{tabular}

B

\begin{tabular}{|lccc|}
\hline Biological Processes & overall & unique & diff. localized \\
protein import \& biogenesis & 1082 & 367 & 89 \\
protein maturation \& folding & 26 & 8 & 2 \\
protein degradation & 26 & 13 & 0 \\
DNA related processes & 30 & 1 & 8 \\
mRNA related processes & 46 & 16 & 7 \\
tRNA related processes & 11 & 8 & 1 \\
ribosome \& translation & 52 & 7 & 2 \\
others of gene expression & 96 & 34 & 3 \\
respiratory chain assembly & 32 & 15 & 1 \\
respiratory chain subunits & 42 & 11 & 0 \\
energy metabolism & 62 & 14 & 2 \\
lipid metabolism & 49 & 6 & 2 \\
nucleotides metabolism & 61 & 5 & 6 \\
amino acids metabolism & 13 & 0 & 1 \\
coenzyme/cofactor biosynthesis & 45 & 2 & 2 \\
Fe/S proteins biosynthesis & 17 & 1 & 1 \\
carriers \& channels & 15 & 0 & 0 \\
regulation \& signaling & 48 & 5 & 1 \\
morphology \& dynamics & 42 & 6 & 13 \\
homeostasis \& stress response & 35 & 3 & 5 \\
developmental processes & 40 & 6 & 2 \\
others & 24 & 5 & 2 \\
unclassified & 105 & 65 & 13 \\
\hline
\end{tabular}

Figure 4. Categorization of the $D$. discoideum mitochondrial protein compendium. (A) $D$.

4 discoideum mitochondrial proteins with homologs in $H$. sapiens or $S$. cerevisiae mitochondrial 5 proteomes, or the complete proteomes of the alphaproteobacteria $R$. prowazekii or $D$. purpureum. 6 (B) The functional categorization of all $D$. discoideum mitochondrial proteins (overall, $\mathrm{n}=1082$ ), 7 those that lack human homologs (unique, $n=367$ ), and those that have human homologs, but 8 homologs were not annotated as mitochondrial proteins (diff. localized, $n=89$ ). 
bioRxiv preprint doi: https://doi.org/10.1101/2021.11.08.467494; this version posted November 8, 2021. The copyright holder for this preprint (which was not certified by peer review) is the author/funder, who has granted bioRxiv a license to display the preprint in perpetuity. It is made available under aCC-BY-NC-ND 4.0 International license.

A

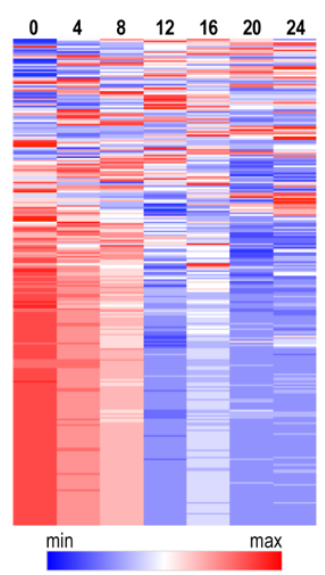

C mt. encoded genes 04812162024

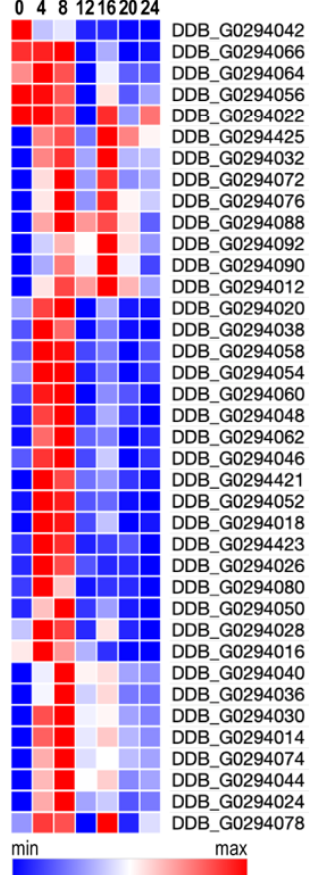

B

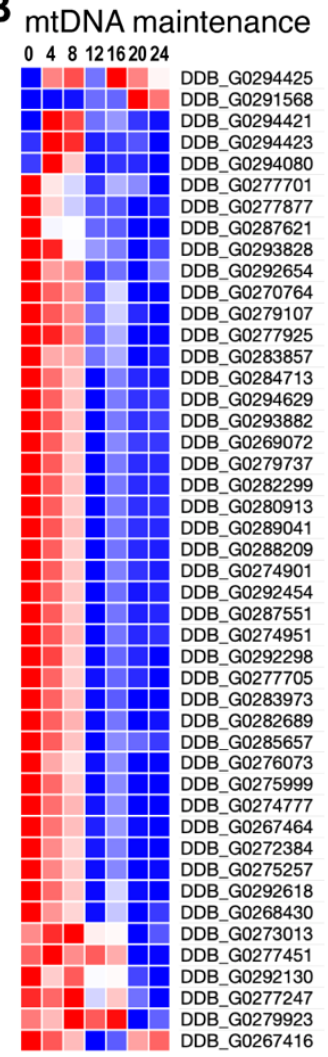

mt. gene expression

04812162024

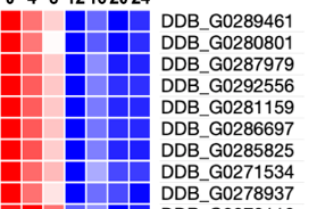

DDB_G0278937
DDB_G0270116

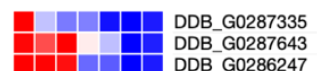

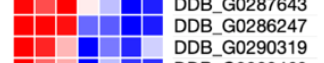

\begin{tabular}{ccc}
\hline & DDB_G0290319 \\
\hline & DDB_G0288469 \\
\hline & DDB_G0270854
\end{tabular}

DDB_G0285125

DDB_G0285693

DDB_G0289215

DDB_G0279261

DDB_G0287997

DDB_G0286199

DDB_G0282387

DDB_G0277013

DDB_G0275919

DDB_G0279835

DDB_G0287775

DDB_G0277611

DDB_G0278079

DDB_G0282961

DDB_G0273417

DDB_G0269880

DDB_G0285575

DDB_G0275823

DDB_G0268662

DDB_G0276449

DDB_G0267648

DDB_G0279659

$\min$

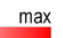

Figure 5. Expression profile of mitochondrial genes during $D$. discoideum development. Heatmaps showing normalized RNA-seq analysis values of $(A)$ all mitochondrial proteins within the compendium and (B-D) specific mitochondrial proteins during the 24-hour starvation-induced development cycle. Each column of heat maps represents the time in hours after developmental induction. Rows are clustered by similarity in gene expression profiles. Boxes outline ETC subunits showing increased expression 4 hours after starvation (solid) and 12 hours after starvation (dashed).

(1)

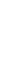

(1)

5

6




\section{Supplemental Tables and Table Legends}

Table S1. D. discoideum homologs of human mitochondrial proteins. Homology assessed by BlastP (expect $<0.001$ and bit-score $>40$ ) using the Human MitoCarta 3.0 as the query and the $D$. discoideum proteome as the subject. Presented alongside are $\mathrm{N}$-terminal sequence predictions retrieved from TarpetP2 (mTP: mitochondrial targeting peptide; SP: signal peptide; OTHER: neither mTP nor SP).

Table S2. Proteomics discovery relative enrichments and relative enrichment ratios. Relative enrichment ratios (columns 2-4) and their respective p-values (columns 5-7) for samples quantified in the proteomic discovery experiment. Relative enrichments from lysate (columns 8 and 9), crude mitochondria (columns 10 and 11), and purified mitochondria (columns 12 and 13) samples. All sample enrichments are calculated from three biological replicates.

Table S3. Proteomics discovery core mitochondrial proteins. $D$. discoideum mitochondrial ETC, OXPHOS, and TCA cycle proteins used for enrichment normalization. Information retrieved from uniprot.org.

Table S4. Proteomics discovery mitochondrial targeting sequence prediction. N-terminal sequence predictions retrieved from TargetP-2.0 for all samples quantified in the proteomic discovery experiment $(n=6892$, columns $1-3)$ and all proteins with an RER $>0.343(n=908$, columns 4-6). (mTP: mitochondrial targeting peptide; SP: signal peptide; OTHER: neither mTP nor SP.)

Table S5. Microscopy validation of relative enrichment ratio cutoff. Localization patterns of proteins used to verify the RER cutoff. A red highlight indicates proteins that were removed from the final mitochondrial compendium while a green highlight indicates proteins that were added to the final mitochondrial compendium based on the protein's localization. Proteins that were unable to be synthesized or transfected and proteins that have homology to a protein within the Human MitoCarta 3.0 are indicated in red and blue, respectively.

Table S6. Classifications of the mitochondrial protein compendium. Biological process classifications were determined by PANTHER biological process or PANTHER family of the $D$. discoideum protein, or of the $H$. sapiens protein. Homologs of the $D$. discoideum proteins were established by HMMER alone (best homolog) or by HMMER and BlastP (D. purpureum whole proteome, $R$. prowazekii whole proteome, $S$. cerevisiae whole proteome and mitoproteome, and $H$. sapiens whole proteome and mitoproteome.

Table S7. Ribosome proteins within the mitochondrial protein compendium. List of all mitochondrial proteins with ribosomal protein classifications according to their protein name and/or PANTHER family.

Table S8. Scaled RNAseq data. The normalized expression profiles for genes encoding mitochondrial proteins (Parikh et al., 2010; Stajdohar et al., 2017) scaled to a mean of 0 and standard deviation of 1. (Groups: biological process categories; t0-24: timepoints in hours within the 24-hour starvation-induced development cycle.) Upregulated genes (log2 fold-change $\geq 1$ ) at specific timepoints are indicated in red.

Table S9. List of oligos used for cloning. Homology to pDM323 used for ligation is underlined. Reverse primers contain an additional base (bolded) to retain reading frame. 